\title{
Mesure des pertes de croissance radiale sur quelques espèces de Pinus dues à deux défoliateurs forestiers. I - Cas de la processionnaire du pin en région méditerranéenne
}

\author{
Nathalie LAURENT-HERVOUËT \\ INRA, Centre de Recherches forestières \\ Station de Zoologie forestière, Ardon, F 45160 Olivet
}

\begin{abstract}
Résumé
L'impact des défoliations dues à la processionnaire du pin (Thatumetopoea pityocampa Schiff.) sur l'accroissement radial des pins a été étudié grâce à la dendrochronologie au Mont Ventoux sur les pins noirs d'Autriche (Pinus nigra Arn. ssp. nigricans Host.) et dans la vallée du Niolo en Corse sur les pins laricio (Pinus nigra Arn. ssp. Iaricio Poiret). Les témoins, non attaqués dans les sites étudiés sont constitués par des cèdres (Cedrus atlantica Manetti) au Ventoux et par des pins maritimes (Pinus pinaster Ait.) en Corse. Une étude dendroclimatologique a permis de connaître l'effet des facteurs climatiques sur l'accroissement afin de l'éliminer et d'étudier uniquement l'effet de la processionnaire.

Au Ventoux, on a pu détecter un cerne nul en 1968 dans deux placettes après la forte attaque de 1966-1967. L'étude des vingt-cinq dernières années montre les faits suivants :

1) l'accroissement est davantage influencé par les attaques de processionnaires que par le climat ;

2) une défoliation à l'automne $\mathrm{n}-1$ et au printemps $\mathrm{n}$ est répercutée sur l'accroissement de l'année $n+1$;

3) la perte globale enregistrée est de 35 p. 100 dans la placette la plus attaquée.

En Corse, les pertes d'accroissement atteignent 20 p. 100 sur l'ensemble des vingt-huit années de l'étude mais les attaques qui n'ont lieu qu'un an sur deux doivent particulièrement compliquer le problème par rapport au Ventoux. Apparemment, seule la première gradation de 1964-1966 s'est répercutée sur l'accroissement. Celle de 1974-1976 ne semble pas avoir eu d'effet, le taux de défoliation observé pendant cette période a toujours été inférieur à 50 p. 100.
\end{abstract}

Mots clés : Pinus, Thaumetopoea pityocampa, pertes d'accroissement, dendrochronologie.

\section{Introduction}

Certaines gradations importantes d'insectes défoliateurs entraînent la défoliation parfois totale des arbres-hôtes. C'est le cas des attaques de la processionnaire du pin (Thaumetopoea pityocampa Schiff.) dans le midi de la France et du Lophyre du pin (Diprion pini L.) en région Centre et dans l'lle-de-France. Il peut alors en résulter de graves conséquences pour la santé et la croissance de l'arbre qui se trouve démuni 
de tout appareil respiratoire et assimilateur. Les pertes de croissance occasionnées par les défoliations dues à la processionnaire du pin ont été étudiées par différentes méthodes :

- soit par défoliation artificielle (Joly, 1959; Polge \& Garros, 1971) ;

- soit après défoliation "naturelle» par les insectes.

Deux méthodes sont possibles : on contrôle les attaques en déposant des colonies de processionnaires sur les arbres à étudier (CADAHIA \& INSUA, 1970) ou bien on étudie les accroissements d'arbres ayant subi des infestations «naturelles». LEMOINE (1977), à l'issue d'une attaque de processionnaires importante mais isolée dans le temps dans les Landes de Gascogne a suivi pendant 5 ans l'accroissement des arbres attaqués et des arbres témoins. Au Mont Ventoux, où les pins noirs d'Autriche sont soumis à des gradations périodiques de la processionnaire, BouchON et TOTH (1971) ont utilisé la dendrochronologie. C'est cette méthode que nous avons choisie pour étudier l'impact des attaques de processionnaires sur l'accroissement radial des pins noirs d'Autriche (Pinus nigra Arn. ssp. nigricans Host.) au Mont Ventoux et des, pins laricio (Pinus nigra Arn. ssp. laricio Poiret) dans les montagnes corses en profitant des données de populations recueillies par le Service forestier au Mont Ventoux depuis 1958 et par l'INRA (GFrI) depuis 1966 en Corse.

Grâce à l'étude des cernes d'accroissement annuel, on peut chiffrer les pertes d'accroissement subies lors des gradations connues et rechercher l'existence des gradations antérieures. Les variations d'accroissement pouvant être influencées par de nombreux facteurs (climat, activités humaines, attaques d'insectes ou de champignons), la connaissance de ces facteurs est indispensable notamment la connaissance des facteurs climatiques qui jouent un rôle prépondérant (FritTs, 1976). Nous avons donc recherché dans les différentes régions étudiées des séries de données météorologiques correspondant aux accroissements observés et aux notations effectućes sur l'importance des populations d'insectes, la connaissance de l'impact des factcurs climatiques constituant un préalable indispensable à l'étude de l'influence des attaques de processionnaires.

\section{Matériel et méthodes}

\subsection{Matériel}

\subsection{Au Ventoux}

L'étude a été effectuée sur les trois placettes de la forêt domaniale de Malaucène contenant respectivement 58,38 et 60 pins, sur lesquels le service forestier a compté les nids à chaque printemps depuis 1958 et établi le nombre moyen de nids par pin et par placette. Les placettes 1 et 2 exposées au S.S.E. sont ćtablies respectivement à 700 et $720 \mathrm{~m}$ d’altitude dans un peuplement clairićré de pins noirs d'Autriche d'environ 90 ans, elles sont régulièrement attaquées, la placette 1 comprend également des cèdres; la placette 3 , à $560 \mathrm{~m}$ d'altitude, située plus à l'ouest est établie dans un peuplement plus régulier; elle subit généralement moins d'attaques. Nous avons prélevé en octobre 1983 vingt carottes de pins noirs d'Autriche à la tarière de Pressler de $5 \mathrm{~mm}$ dans chaque placette et vingt carottes de cèdres dans la placette 1 ; cette essence non attaquée nous servant de témoin, considérant que ces cèdres installés 
récemment dans cette partie du Ventoux sont soumis uniquement aux phénomènes généraux et ne subissent pas d'accident particulier à l'espèce.

Pour la connaissance du climat, nous avons pu travailler sur les relevés des précipitations et températures mensuelles enregistrées depuis 1972 par le S.T.E.F.C.E. (Service Technique d'Etude des Facteurs Climatiques et de l'Environnement) de l'INRA d'Avignon dans la combe de Bramefam à $710 \mathrm{~m}$, très près des placettes d'échantillonnage et également sur les relevés pluviométriques mensuels effectués de 1958 à 1975 au village de Malaucène à $300 \mathrm{~m}$ d'altitude. Les informations recueillies de 1972 à 1975 dans les deux sites nous ont permis d'établir une régression linéaire entre les précipitations de Bramefam et celles de Malaucène et de calculer ainsi une estimation de la pluviométrie mensuelle de Bramefam de 1958 à 1972.

\subsection{En Corse}

Les placettes d'étude ont été choisies dans la vallée d'altitude du Niolo afin de bénéficier des indices d'infestation de processionnaires calculés par zones et sous zones après enquête depuis 1966 par l'INRA (GERI, 1971, 1975, 1980, 1983). Différents indices ont été calculés, nous avons utilisé l'indice suivant :

0 Aucun nid

1 Présence d'un ou de quelques nids dans le peuplement

2 Infestation faible (moins d'un nid par pin)

3 Un nid par pin environ

4 Entre 1 et 5 colonies par pin

5 Entre 5 et 10 colonies par pin en moyenne

6 Entre 10 et 20 colonies par pin en moyenne

7 Plus de 20 colonies par pin en moyenne

Les parcelles «PK 51 » et «Tilerga » respectivement à 950 et 920 m d'altitude et en exposition E.N.E. et E.S.E. sont situées dans des peuplements très clairiérés qui occupent d'anciennes terres de culture recolonisées depuis 80 ans par la forêt. La parcelle «Casamaccioli » exposée au N.O. à $1050 \mathrm{~m}$ est peuplée par une futaie assez dense d'arbres âgés (environ 150 ans). Dans chaque parcelle, 20 carottes de pins laricio ont été prélevées en juin 1983, le témoin a été constitué de 20 carottes de pins maritimes présents dans la parcelle de Tilerga, essence très peu attaquée en Corse au-dessus de $900 \mathrm{~m}$.

On a utilisé les informations climatiques provenant des différents postes météo de la vallée, implantés soit par l'O.N.F. (Popaja à $1000 \mathrm{~m}$ ), soit par le S.T.E.F.C.E. (Frascajo, $980 \mathrm{~m})$ et PK $51(920 \mathrm{~m})$. Nous avons ainsi pu disposer de la pluviométrie mensuelle de 1955 à 1981 et des températures mensuelles de 1955 à 1966 et de 1974 à 1981 .

\subsection{Méthodes}

\subsection{Synchronisation (Munaut, 1978 ; TrEnard, 1982)}

Un graphique des accroissements annuels en fonction du temps est construit pour chaque carotte; dans chaque placette et pour chaque essence on procède à la synchronisation : on admet d'après l'hypothèse de Douglass en 1937 (in Trenard, 1982) que les arbres d'une même essence en un même lieu réagissent de la même 
façon aux phénomènes extérieurs; les courbes individuelles devraient donc présenter des variations identiques et synchrones. Toutefois, on a souvent le phénomène suivant (fig. 1) :

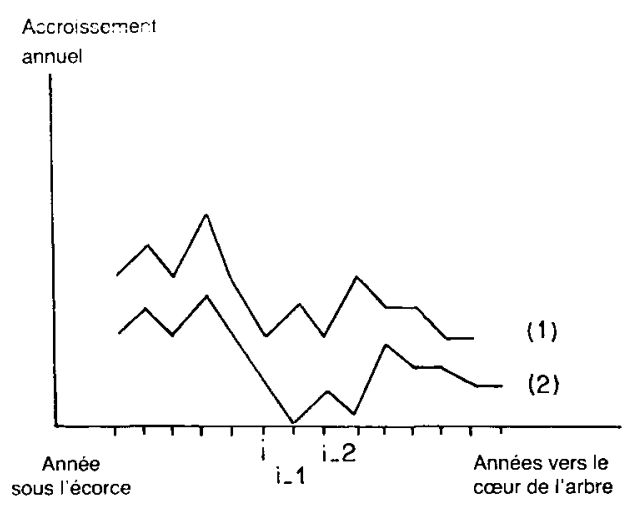

FIG. 1

Exemple de synchronisation.

Synchronisation example.

En remontant dans le temps, les courbes sont synchrones jusqu'à une année i, pour les années antérieures elles sont décalées d'une annće l'une par rapport à l'autre. Il peut y avoir deux explications.

- Il manque le cerne de l'année i ou de lannée i - 1 sur la courbe (1). L'absence de ce cerne peut provenir :

- d'un oubli lors de la lecture;

- d'un cerne très mince et peu marqué qui a été lu avec un autre cerne;

- d'une croissance radiale nulle.

- Sur la courbe (2), il y a un cerne double l'année i ou i - 1 correspondant soit à une erreur de lecture à cause d'une inclusion de résine par exemple, soit pour une même annce de végétation à l'élaboration de deux assises de bois initial et deux assises de bois final.

Un examen minutieux de chaque carotte et la comparaison avec les courbes des autres arbres permet généralement de choisir l'une de ces hypothèses. Puis on construit une courbe standard; elle est constituée par la moyenne des accroissements annuels des arbres ayant les courbes les mieux synchronisées entre elles. Ce sont souvent de gros arbres, afin de diminuer les erreurs dues aux lectures de cernes. L'intérêt de cette courbe est de faire ressortir les accidents collectifs en éliminant les arbres (1/4 de l'effectif environ) qui présentent de trop fortes variations individuelles. 


\subsection{Analyse statistique}

L'analyse en régression multiple et l'analyse en composantes principales ont été utilisées pour rendre compte des relations accroissement-climat et accroissementattaque.

\subsection{L'analyse en régression multiple}

Elle permet d'expliciter une variable (l'accroissement annuel) en fonction de plusieurs variables explicatives (variables climatiques et variables d'infestation) suivant le modèle :

avec $y:$ variable expliquée

$$
y=\sum_{i=1}^{n} \alpha_{i} x_{i}+C+1
$$

$x_{i}:$ ième variable explicative

$\alpha_{\mathrm{i}}$ : coefficient de régression de la ième variable explicative

C : constante

1 : résidu (variable aléatoire)

Nous avons utilisé un programme de régression multiple progressive où les variables explicatives sont introduites une à une par palier (BACHACOU et al., 1981). Compte tenu de de la bibliographie (FritTs, 1976), nous avons supposé que le climat était le facteur prépondérant de l'accroissement et fait d'abord plusieurs régressions multiples progressives avec toutes les moyennes mensuelles de températures et les moyennes mensuelles, bimensuelles et trimestrielles de précipitations correspondant à l'année de végétation (septembre $n-1$ à août $n$ ), ce qui a permis de sélectionner les variables importantes qui seules ont été finalement conservées dans l'équation de régression (LAURENT-Hervouët, 1984).

Pour la Corse comme au Ventoux, l'étude a été effectuée sur l'ensemble des années pour lequel on possède des données climatologiques puis au Ventoux en excluant les années succédant à de fortes gradations pour éliminer lincidence des attaques de processionnaires. Dans un deuxième temps, on a étudié l'effet des attaques sur l'accroissement en choisissant comme variables explicatives de l'accroissement de l'année $n$, les populations observées au printemps des années $n, n-1, n-2, n-3$ et les variables climatiques sélectionnées précédemment. Puis nous avons étudié pour le Ventoux, l'effet des populations des années $n, n-1, n-2, n-3$, sur les écarts $P_{i}$ entre les valeurs observées des accroissements $y_{i}$ obs et leur estimation par le modèle de régression $\hat{y}_{\mathrm{i}}$ th avec les facteurs climatiques établi au cours des années sans. attaque. On a alors pu calculer la perte d'accroissement radial $P_{j}$ :

$$
P_{j}=y_{j} \text { obs }-\hat{y}_{j} \text { th }
$$

où $y_{j}$ obs est l'accroissement radial observé l'année $j$ et $\hat{y}_{j}$ th l'estimation de l'accroissement compte tenu du climat avec : lectionnée i.

$\hat{y}_{j}$ th $=\sum_{i=1}^{n} \alpha_{i j} x_{i j}+C$ avec $x_{i j}$ valeur pour l'année $j$ de la variable climatique sé-

Pour le Mont Ventoux, l'équation de la régression est calculée sur les années sans attaque; en Corse, elle est calculée à partir des pins maritimes. 


\subsection{L'analyse en composantes principales}

L'analyse en composantes principales permet de réduire le nuage multidimensionnel représentant les données à un nombre limité d'axes : les premiers axes principaux (Cailliez \& Pages, 1976).

Cette méthode a été utilisée pour analyser les données recueillies arbre par arbre dans une placette en Corse. Dans cette placette (PK 51) les principales caractéristiques dendrométriques de 11 pins ont été notées depuis 1972 ainsi que le nombre de nids de processionnaires à chaque printemps et un indice de défoliation quoi que ce dernier ait été relevé de façon moins systématique. L'analyse en composantes principales a porté sur les variables d'accroissements individuels de 1972 à 1982 et les nombres de nids des années paires de 1972 à 1982.

On peut travailler sur deux types de matrices :

\section{MATRICE A :}

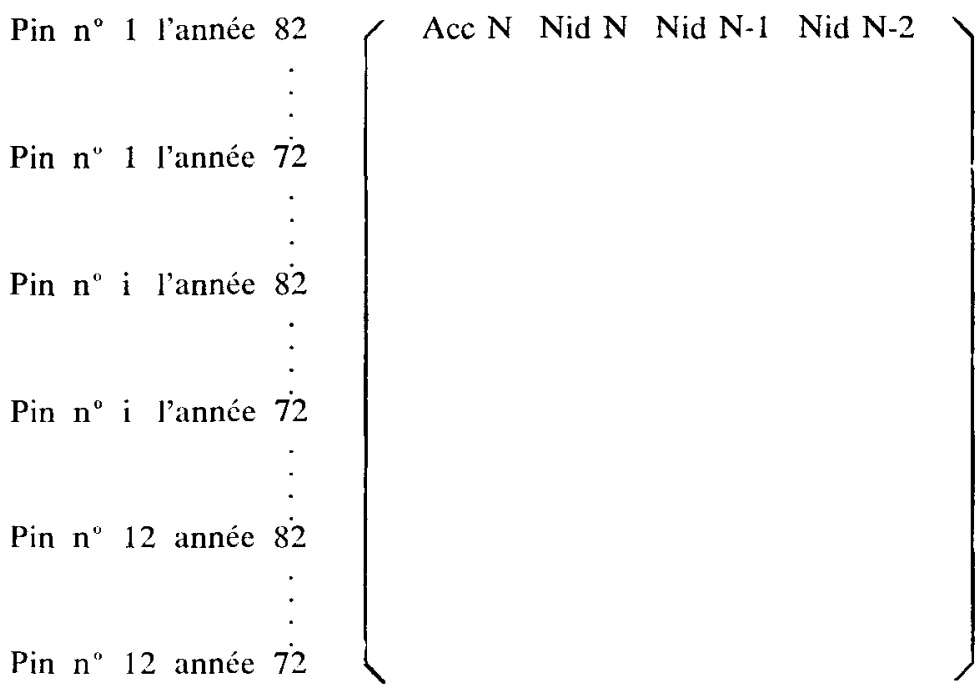

MATRICE B :

$\left.\begin{array}{ccc}\text { Pin } n^{\circ} & 1 \\ \vdots & \\ \text { Pin } n^{\circ} & i \\ \vdots & \\ \text { Pin } n^{\circ} & 12\end{array}\right)$

Les résultats de l'analyse avec la matrice $\mathrm{A}$ étant peu démonstratifs en raison de l'absence de processionnaires un an sur deux, seuls les résultats de l'analyse avec la matrice B seront exposés. Nous avons de plus introduit en variables supplémentaires c'est-à-dire ne participant pas au calcul des axes les variables dendrométriques 
pour chaque arbre : hauteur, circonférence, surface terrière de la placette située autour de chaque arbre et mesurée au relascope de Bitterlich ainsi que le nombre total de nids supportés de 1972 à 1982.

\section{Rappels sur la biologie de la processionnaire}

La processionnaire du pin (Thaumetopoea pityocampa Schiff.) est un lépidoptère Thaumetopoeidae. Les aufs sont pondus entre le 15 juillet et le 15 août et éclosent entre août et septembre, les chenilles défoliatrices s`alimentent la nuit et subissent quatre mues. Leurs dégâts deviennent importants à partir du troisième stade larvaire ; au quatrième stade, à l'automne, elles tissent de grosses bourses de soie qui selon Demolin (1965, 1969 b) jouent le rôle de volants thermiques et de radiateurs solaires constituant leur protection durant la période hivernale.

Après leur évolution larvaire, de mars à juin, suivant les régions, les chenilles descendent en procession des arbres, senfouissent dans le sol, tissent un cocon et $y$ subissent la nymphose. La sortie des adultes dans les régions d'altitude du midi de la France a lieu du 15 juillet au 15 août. Les papillons s'accouplent et pondent dans les 48 heures puis meurent. Le choix des arbres par les femelles ainsi que les preferendums thermiques des chenilles (Demolın, $1969 \mathrm{a}, 1971,1974$ ) ont des conséquences sur la répartition des attaques dans le peuplement; les peuplements clairiérés, les lisières et en montagne les pins en exposition sud sont les plus attaqués (GERI, 1980, 1983). Le cycle biologique peut varier d'une région à l'autre : au Mont Ventoux, où l'habitat des chenilles, limité à $900 \mathrm{~m}$, est plus chaud que dans les forêts d'altitude de Corse qui s'étendent jusqu’à 1500-1-600 m (Geri \& Millier, 1985), le cycle est annuel. Dans les montagnes corses, au-dessus de $900 \mathrm{~m}$, le développement est plus lent, l'insecte ne peut pas effectuer la totalité de son cycle biologique en une année, celui-ci devient bisannuel (Demolin, 1971) ; suivant les vallées les vols d'adultes ont lieu les années paires (Vizzavone, Verghello, Restonica, Asco) ou impaires (Niolo, Aitone).

\section{Résultats obtenus au Ventoux}

\subsection{Courbes standard}

Les trois courbes standard (fig. 2) obtenues après avoir synchronisé les pins de chaque placette sont synchrones entre elles et synchrones avec la courbe des cèdres, sauf de 1967 à 1974 , période pendant laquelle la synchronisation a permis de détecter la présence de cernes nuls en 1967 pour les pins de la placette 2 et en 1968 pour les pins des placettes 1 et 2 . La courbe des pins de la placetie 3 montre vers cette époque une baisse d'accroissement mais pas de cerne nul. La comparaison des courbes d'accroissement avec la courbe d'attaques de processionnaires montre que la période pendant laquelle on observe des cernes nuls et pour laquelle les courbes pins-cèdres ne sont pas synchrones correspond à la période d'infestation maximale.

Le cerne nul observé en 1968 dans les placettes 1 et 2 semble être la conséquence de la très forte attaque de 1967, la placette 3 moins attaquée a subi une 

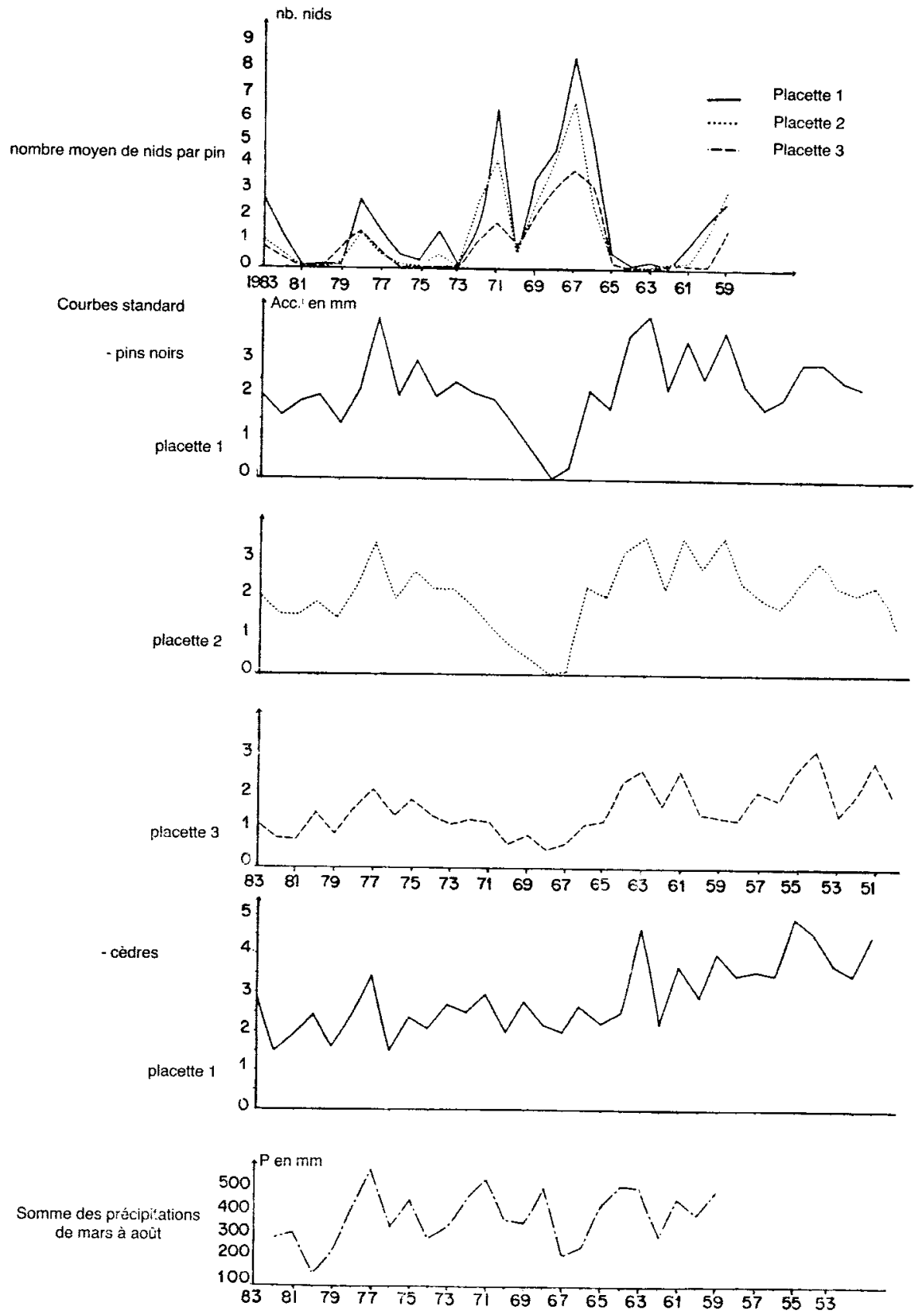

FIG. 2

Comparaison des courbes standard obtenues au Ventoux avec l'évolution des populations de processionnaires et les précipiataions de la saison de végétation.

Comparison between the standard curves on «Mont Ventoux" and the evolution of the pine processionary moth populations and the precipitations of the growing season. 
chute d'accroissement moins brutale. On assiste ensuite, alors que le nombre moyen de nids diminue, à une récupération difficile des pins qui dure 5 ans.

La comparaison des courbes d'accroissement avec la somme des précipitations de mars à août montre une certaine concordance en dehors de la période 1967-1974 pour les pins et pendant toute la durée 1957-1983 pour les cèdres. Les accroissements des pins sont très liés à la processionnaire du pin pendant les périodes d'infestation, proportionnellement aux attaques subies : les placettes 1 et 2 très attaquées présentent des cernes nuls, la placette 3, la moins attaquée, présente seulement une baisse d'accroissement. En dehors des grandes périodes d'infestation, l'accroissement des pins est semblable à celui des cèdres et est lié aux précipitations. Il n'apparaît pas de relation entre les accroissements et les dates d'éclaircies, l'action de l'homme au Ventoux ne se répercute pas directement sur l'accroissement radial.

\subsection{Influence du climat sur l'accroissement}

Le tableau 1 montre que pour l'ensemble des années, le meilleur ajustement est donné par les cèdres et la placette de pins la moins attaquée, cependant les coefficients de corrélation et les tests $F$ montrent que le meilleur ajustement de l'équation de régression aux données est obtenu pour les années hors attaque, c'est pourquoi nous prendrons par la suite les variables et l'équation de régression obtenues hors attaque. Ceci confirme lincidence des attaques de processionnaires sur les accroissements des pins et la nécessité d'en apprécier l'importance compte tenu des variables climatiques.

\section{TABleau 1}

Variables climatiques influençant l'accroissement radial au Ventoux. Climatic variables influencing the radial growth at Ventoux.

1 a : Variables sélectionnées sur l'ensemble des années.

1 a: Selected variables on all the years.

\begin{tabular}{|c|c|c|c|}
\hline & Parcelle 1 & Parcelle 2 & Parcelle 3 \\
\hline Pins & $\begin{array}{l}\mathrm{P} \text { (mars + avril) } \\
\mathrm{P} \text { (sept. }+ \text { oct.) } \\
\mathrm{P} \text { déc. } \\
\mathrm{R}=0,635 \\
\mathrm{~F}=3,602^{*}\end{array}$ & $\begin{array}{l}\mathrm{P} \text { (mars + avril) } \\
\mathrm{P} \text { (sept. }+ \text { oct.) } \\
\mathrm{P} \text { déc. } \\
\mathrm{R}=0,617 \\
\mathrm{~F}=3,287\end{array}$ & $\begin{array}{l}P \text { (mars + avril) } \\
P \text { (sept. }+ \text { oct.) } \\
P \text { déc. } \\
R=0,698 \\
F=5,059 *\end{array}$ \\
\hline$\overline{\text { Cèdres }}$ & $\begin{array}{l}\mathrm{P} \text { (nov. }+ \text { déc. }+ \text { janv. }) \\
\mathrm{P} \text { (mai }+ \text { juin) } \\
\mathrm{P} \text { (sept. }+ \text { oct. }) \\
\mathrm{R}=4,685^{*}\end{array}$ & & \\
\hline
\end{tabular}

$1 b$ : Variables sélectionnées sur les années hors-attaque.

$f b$ : Selected variables on the years without outbreaks.

\begin{tabular}{|c|c|c|c|}
\hline & Parcelle 1 & Parcelle 2 & Parcelle 3 \\
\hline Pins & $\begin{array}{l}P \text { janv. } \\
P \text { avril } \\
P \text { juin } \\
P \text { (sept. + oct.) } \\
R=0,943 \\
F=16,022^{*}\end{array}$ & $\begin{array}{l}P \text { janv. } \\
P \text { avril } \\
P \text { juin } \\
P \text { (sept. + oct.) } \\
R=0,947 \\
F=17,335^{* *}\end{array}$ & $\begin{array}{l}P \text { janv. } \\
P \text { juin } \\
P \text { juillet } \\
P \text { (nov. + déc.) } \\
R=0,949 \\
F=18,225^{*}\end{array}$ \\
\hline
\end{tabular}


Les mois importants semblent être ceux du début de la saison de végétation (mars, avril, juin), ce qui correspond aux résultats de l'étude graphique (fig. 1) mais également les mois de l'automne-hiver de l'année précédente (septembre, octobre, décembre) la pluviosité de cette période permet la reconstitution des réserves hydriques du sol, tandis que les précipitations du printemps évitent un stress hydrique en début de saison de végétation.

\subsection{Influence de la processionnaire sur l'accroissement}

\subsection{Etude sur lacoroissement}

Les résultats des régressions multiples progressives ayant comme variable expliquée l'accroissement de l'annće $n$ et comme variables explicatives les variables climatiques précédemment sélectionnées et les données de population de processionnaires par placette sont présentés pour la placette 1 tableau 2. Des résultats très voisins sont obtenus pour les pins des autres placettes. La première variable introduite est le nombre de nids de l'année antérieure, les pluies du printemps (mars, avril) viennent ensuite, suivies des attaques des autres années. Les attaques présentent avec l'accroissement un coefficient de régression négatif alors que pour les pluies le coefficient est positif. Il apparaît donc, contrairement à notre hypothèse que ce sont les attaques de processionnaires et non le climat qui influencent le plus l'accroissement des pins au Ventoux.

\section{Tableau 2}

Influence des populations de processionnaires et des principaux facteurs climatiques sur l'accroissement radial des pins noirs de la placette 1 au Ventoux. Principaux résultats de la régression multiple progressive.

Influence of the pine processionary moth populations and the main ctimatic factors on the radial growth on "Mont Ventoux» black pines (parcel 1).

Equation finale :

Main results of the stepwise regression.

$$
\begin{aligned}
& \hat{y}=0,86+0,99 \cdot 10^{-3} \text { P Déc. }+0,16.10-3 \mathrm{P}(\text { Sept. }+ \text { Oct. })+0,69.10 \text { : } \mathrm{P} \text { (Mars + Avril) } \\
& -0,12 \mathrm{Nid} N-0,90 \cdot 10^{1} \mathrm{Nid}(\mathrm{N}-1)-0,15 \text { Nid }(\mathrm{N}-2)-0,45 \cdot 10^{-1} \text { Nid } \\
& (\mathrm{N}-3) \text {. }
\end{aligned}
$$

\begin{tabular}{|c|c|c|c|c|}
\hline $\mathrm{N}^{\prime \prime}$ de palier & Variable introduite & ra & $\mathrm{R}$ & $\mathrm{F}$ \\
\hline 1 & Nid $N-1$ & $-0,31^{*}$ & 0,685 & $15.84 *$ \\
\hline 2 & P (Mars + Avril) & $0,74.10^{3}$ & 0.746 & $10,65^{*: *}$ \\
\hline 3 & Nid $N$ & $-0,11$ & 0,779 & $8,22 *$ \\
\hline 4 & Nid $N-2$ & $-0,94.10 \quad 1$ & 0,800 & $6,68 * *$ \\
\hline 5 & P Déc. & $0,87.10 \cdots 3$ & 0.825 & $5.97 *$ \\
\hline 6 & $\mathrm{I}^{\prime}($ Sept. + Oct $)$ & $0,14.10 \cdots$ & 0,830 & $4,80 * *$ \\
\hline 7 & Nid $N-3$ & $0.45 \cdot 10^{-1}$ & 0,833 & $3.88 \%$ \\
\hline
\end{tabular}

Symboles utilisés :

$\alpha$ : coefficient de régression de la variable à l"introduction.

$\mathrm{R}$ : coefficient de corrélation multiple.

$F$ : test gobal d'ajustement. 
Un calcul identique a été effectué pour les cèdres. II ne montre, bien entendu, aucune incidence des attaques de processionnaires et ce sont les précipitations qui conditionnent le plus l'accroissement. La perte de croissance un an après l'attaque sur les pins noirs d'Autriche du Ventoux a déjà été notée par Bouchon \& ToTH (1971). Elle s'explique si l'on considère que les conifères et les feuillus de la zone tempérée utilisent des réserves photosynthétisées et stockées l'année précédente pour élaborer le bois de printemps et effectuer leur première croissance en hauteur (KuI.MAN, 1971).

\subsection{Etude de la différence entre valeur observée et valeur estimée de l'accroissement}

On étudie comment le terme $P_{j}=y_{i}$ obs $-\hat{y}_{i}$ th qui représente la part de l'accroissement indépendante du climat est influencée par les attaques. Les résultats sont présentés pour la placette 1 tableau 3, ces résultats sont concordants pour les trois placettes. Dans tous les cas, les variables explicatives sélectionnées en premier sont les nombres de nids des années précédentes et en particulier le nombre de nids de l'année $\mathrm{n}-1$, le nombre de nids de l'année ( $\mathrm{Nid} \mathrm{N}$ ) est toujours très peu explicatif. La valeur élevée des tests d'ajustement global et des coefficients de corrélation multiple montrent l'étroite corrélation de $\mathbf{P}_{\mathrm{i}}$ avec les attaques ce qui justifie l'utilisation de ce terme comme un estimateur des pertes d'accroissement annuelles.

\section{TABleaU 3}

Influence des populations de processionnaires sur le terme : $P_{i}=y_{i}$ obs $-\hat{y}_{i} t h$. Influence of the pine processionary moth populations on the term $: P_{i}=y_{i}$ obs $-\hat{y}_{i} t h$.

Equation finale :

$\hat{\mathrm{p}}=0,55-0,28 \cdot 10^{-1}$ Nid $\mathrm{N}-0,62^{* *} \mathrm{Nid}(\mathrm{N}-1)+0,12 \mathrm{Nid}(\mathrm{N}-2)-0.30^{*} \mathrm{Nid}$ $(\mathrm{N}-3)$.

\begin{tabular}{c|c|c|c|c}
\hline \hline $\mathrm{N}^{*}$ de palier & Variable introduite & $\alpha$ & $\mathrm{R}$ & $\mathrm{F}$ \\
\hline 1 & Nid N-1 & $-0,61^{* * *}$ & 0,751 & $23,29^{* *}$ \\
2 & Nid N-3 & $-0,25$ & 0,808 & $15,94^{* *}$ \\
3 & Nid N-2 & 0,12 & 0,816 & $10,65^{* *}$ \\
4 & Nid N & $-0,28.10^{-1}$ & 0,817 & $7,52^{* * *}$ \\
\hline
\end{tabular}

Symboles utilisés :

$\alpha$ : coefficient de régression de la variable à J'introduction.

$\mathbf{R}$ : coefficient de corrélation multiple.

F : test gobal d'ajustement.

\subsection{Calcul des pertes d'accroissement}

Les pertes daccroissement annuelles sont représentées sur la figure 3 pour le; trois placettes comparativement au nombre moyen de nids par pin. Le graphique met en évidence l'incidence des infestations de processionnaires de l'année précédente, les pertes $P_{j}$ sont toujours significatives à la suite des années d'attaque. Le 

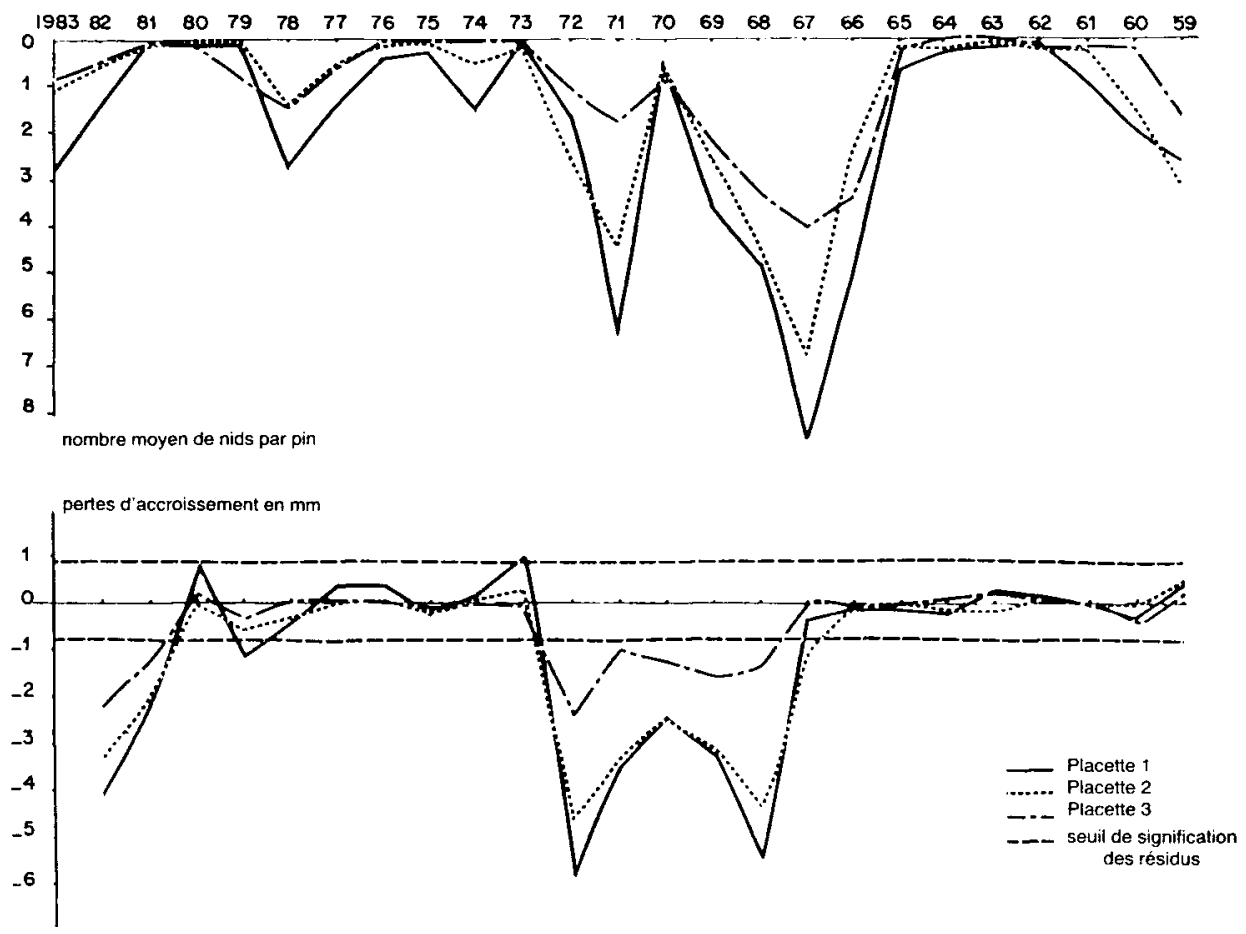

FIri, 3

Comparaison entre les pertes daccroissement madial annuelles ef le nombre moyen de nids par pin au Ventoux.

Comparison between anmual radial growth losses and the mean number of hests per pine on "Mont Ventoux».

terme $P_{i}$ est rarement positif et significatif, c'est le cas de 1973 pour la placette 1 . Il est possible que l'abondante pulviosité estivale de 1973 ait favorisé l'accroissement att-delà des limites prévues par le modèle climatique: mais ce fait peut également résulter d'une reprise de l'accroissement supérieure à la normale après de fortes. attaques, phénomène déjà observé par Polge \& Garros (1971) et WebB \& WickmaN (1978). En effet, cette reprise s'observe surtout dans les placettes les plus attaquées.

Sur plusieurs années, les pertes d'accroissement radial peuvent être calculées de la même façon. On a pour $n$ années :

$$
\begin{aligned}
& P_{11}=\mid \sum_{i=1}^{n} y_{i} \text { obs }-\sum_{i=1}^{n} \hat{y}_{i} \text { th } \mid \text { soit en pourcentage d'épaisseur de cerne } \\
& P^{\prime}{ }_{n}=\left(1-\frac{\sum_{i=1}^{n} y_{i} \text { obs }}{\sum_{i}^{n} \hat{y}_{i} \text { th }}\right) \times 100
\end{aligned}
$$


La signification de la quantité $P_{n}$ est testée par le test de comparaison de deux séries de moyennes appariées (ici l'une théorique, l'autre observée) pour des petits échantillons (Schwartz, 1969). Les calculs ont été détaillés en fonction de plusieurs périodes correspondant d'une part aux gradations (1979 à 1982 et 1968 à 1972) et d'autre part à la période entière pour laquelle nous possédons des données complètes (1959 à 1982) et pendant laquelle se sont succédées des phases de gradation et d'intergradation. Sur la période 1979-1982 qui a suivi une remontée de populations à partir de 1976 puis un traitement d'une grande partie de cette face du Ventoux en 1979, il apparaît une réduction d'accroissement qui n'est pas significative sans doute à cause du très faible nombre de degrés de liberté. Par contre, la perte de croissance est très significative après la gradation de 1967 ainsi que sur l'ensemble des années. Pour la période 1968-1972, les pertes d'accroissement en pourcentage d'épaisseur de cerne sont respectivement pour les trois placettes de 76,5, 82,6 et 63 p. 100. Durant la période 1959-1982, les pertes globales sont respectivement de $33,3,34,3$ et 25,7 p. 100 .

\section{Résultats obtenus en Corse}

\subsection{Etude des courbes standard}

Les courbes standard des pins laricio obtenues dans les trois placettes, présentées figure 4 , sont synchrones bien que celle de la placette Casamaccioli constituée de très vieux arbres (150 à 200 ans) soit beaucoup plus aplatie. Les courbes de PK 51 et de Tilerga, assez concordantes avec la courbe de pins maritimes, divergent de 1964 à 1970. Cette divergence correspond à une période de forte infestation dans le Niolo (gradation de 1964-1966; GERI, 1971), si l'on tient compte d'une certaine période de récupération après les attaques; l'incidence d'une autre période d'attaque (1972 à 1976) qui paraît s'être traduite par une défoliation plus limitée dans les placettes n'apparaît pas nettement. En revanche une autre phase de réduction des accroissements sur le laricio s'observe de 1950 à 1954, elle correspondrait également à de fortes attaques de processionnaires observées par les habitants de la vallée. La courbe des pins maritimes comme celle des pins laricio en dehors des périodes d'attaque suit assez bien la courbe des précipitations. Il apparaît donc que le pin maritime est un bon témoin des pins laricio et que les défoliations dues à la processionnaire peuvent avoir un rôle négatif sur l'accroissement.

\subsection{Influence du climat sur l'accroissement}

Possédant des donnćes de population moins précises qu'au Ventoux (indices d'infestation au lieu du nombre moyen de nids par pin) et les années sans attaque semblant plus difficiles à préciser qu'au Ventoux en raison du cycle bisannuel de la processionnaire, nous n'avons pas déterminé arbitrairement de période hors attaque mais effectué les analyses en régression multiple pour sélectionner les variables climatiques sur l'ensemble des années. Les résultats de cette sélection montrent que ce sont les précipitations de mars, avril, mai et juin qui sont très importantes dans le Niolo : elles ćvitent le stress hydrique en début de saison de végétation et ont une influence positive sur l'accroissement. 
Acc. en mm
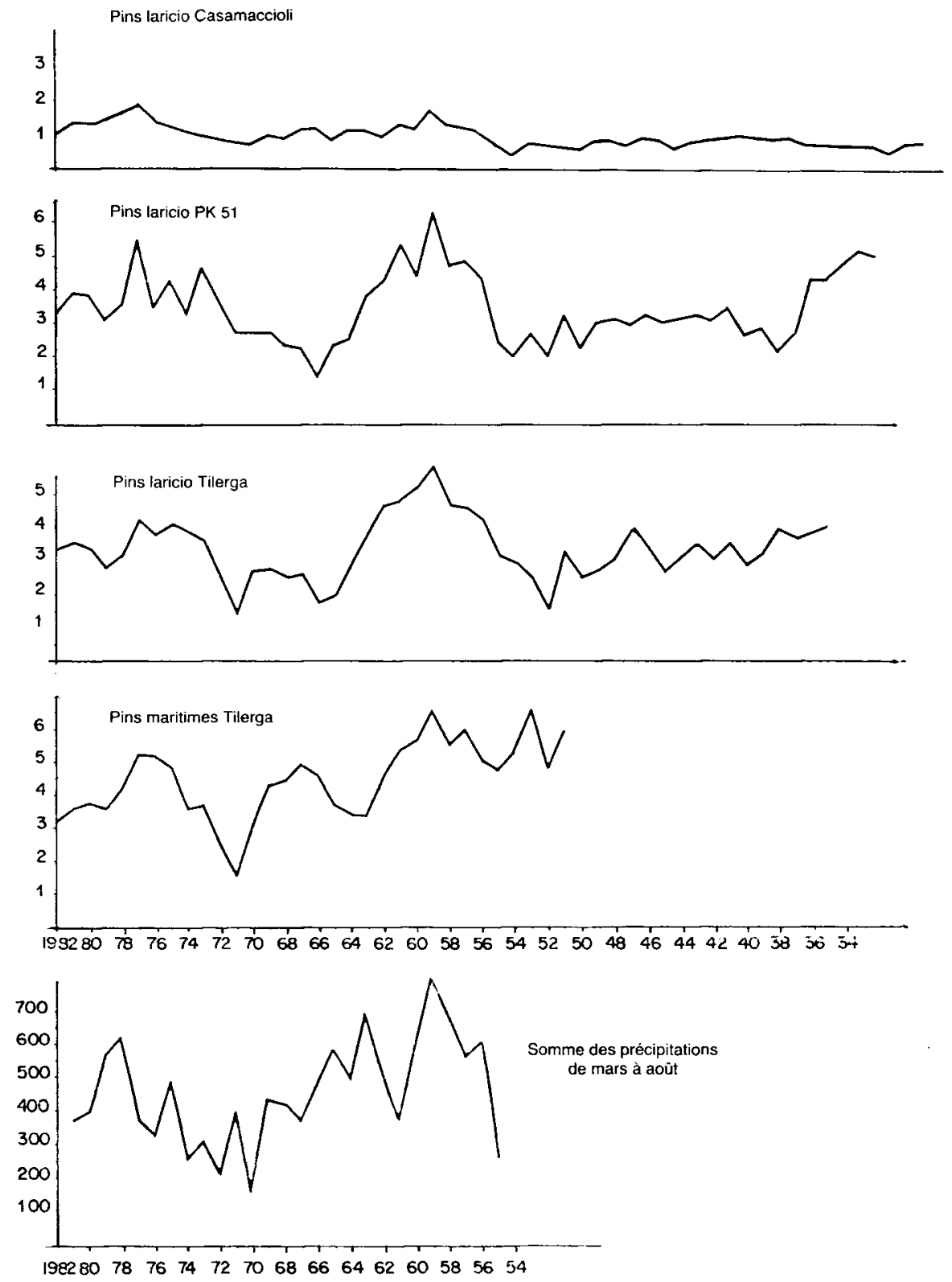

FIG. 4

Comparaison des courbes standard obtenues en Corse avec les précipitations de la saison de végétation.

Comparison between standard curves in Corsica and the precipitations of the growing season. 


\subsection{Influence des attaques de processionnaires}

\subsection{Etude sur les moyennes d'accroissements}

L'étude en régression multiplie des accroissements en fonction des variables climatiques et des indices d'infestation ne met en évidence aucun effet significatif des attaques. On peut considérer différents facteurs qui mettent en cause le bien fondé de cette analyse :

- le cycle bisannuel de la processionnaire entraîne l'introduction d'une infestation nulle un an sur deux;

- la prise en compte des attaques des trois années antérieures limite le calcul aux années 1969 à 1982 (les enquêtes n'ayant commencé qu'en 1966) : les années de forte gradation, confirmées par l'étude de synchronisation, ne sont pas prises en considération durant cette courte période. Dans ces conditions, nous avons testé l'influence des attaques sur l'accroissement des pins laricio par une méthode indirecte grâce aux pins maritimes témoins. On suppose que la valeur de laccroissement calculée sur les pins maritimes à partir de la régression avec les facteurs climatiques est proche de l'accroissement que devrait avoir le pin laricio s'il n'y avait pas d'attaque. Cette valeur estimée est comparée par un test $t$ aux accroissements observés pour le laricio dans les deux placettes ayant subi de fortes attaques (PK 51 et Tilerga) (tabl. 4).

\section{TABLEAU 4}

Equation de la régression : accroissement du maritime en fonction des facteurs climatiques. Valeur et signification de la quantité : $P_{i}=y_{i}$ obs $-\hat{y}_{i}$ th.

Equation of the regression : cluster pine radial growth according to the climatic factors. Equation : Value and significance of the term $P_{i}=y_{i}$ obs $-\hat{y}_{i} t$.

$\begin{aligned} \hat{y}= & 0,27 \cdot 10^{-3} \mathbf{P}(\text { Sept. }+ \text { Oct. })^{* *}+0,16 \cdot 10^{-3 *} \mathbf{P}(\text { Fév. }+ \text { Mars }+ \text { Avril })^{*}+0,811 \cdot 10^{-3} \\ & \mathbf{P} \text { (Mai + Juin + Juil. })^{* *}+0,15 \cdot 10^{-1 *} .\end{aligned}$

Au dernier palier, $\mathrm{R}=0,771$

$$
\mathrm{F}=10,720 * *
$$

Valeurs annuelles de la différence $\mathrm{P}_{\mathrm{i}}=\mathrm{y}_{\mathrm{i}}$ obs $-\hat{\mathrm{y}}_{\mathrm{i}}$ th

\begin{tabular}{|c|c|c|c|c|c|}
\hline Année & PK 51 & Tilerga & Année & PK 51 & Tilerga \\
\hline 1981 & $-0,873$ & $-1,283$ & $1968 \ldots$ & $-1,707 * *$ & $-1,527 * *$ \\
\hline $1980 \ldots$ & 0,113 & $-0,427$ & $1967 \ldots$ & $-3,280^{* *}$ & $-2,930 * *$ \\
\hline $1979 \ldots$ & $-0,614$ & 0,894 & $1966 \ldots$ & $-3,451 * *$ & $-3,081^{* * *}$ \\
\hline $1978 \ldots$ & $-0,729$ & $-1,139$ & $1965 \ldots$ & $-2,030^{*}$ & $-2,490^{* *}$ \\
\hline $1977 \ldots$ & 0,061 & $-1,189$ & $1964 \ldots$ & $-1,470$ & $-1,080$ \\
\hline $1976 \ldots$ & $-0,006$ & 0,284 & $1963 \ldots$ & $-0,538$ & $-0,628$ \\
\hline $1975 \ldots$ & 0,519 & 0,289 & $1962 \ldots$ & $-0,455$ & $-0,165$ \\
\hline 1974 & $-0,181$ & 0,469 & $1961 \ldots$ & 0,298 & $-0,212$ \\
\hline 1973 & 1,122 & 0,022 & $1960 \ldots$ & $-0,645$ & 0,005 \\
\hline 1972 & 0,668 & $-0,372$ & $1959 \ldots$ & 0,596 & 0,136 \\
\hline 1971 & $-0,467$ & $-1,737^{*}:$ & $1958 \ldots$ & $-0,408$ & $-0,438$ \\
\hline 1970 & $-0,162$ & $-0,112$ & $1957 \ldots$ & $-0,895$ & $-1,125$ \\
\hline 1969 & $-1,353$ & $-1,283$ & $1956 \ldots$ & $-0,403$ & $-0,603$ \\
\hline
\end{tabular}


Les différences (valeur observée-valeur estimée) sont négatives et significatives pour les années 1965-1966-1967-1968 correspondant à une période de forte gradation mise en évidence par l'étude démographique (GERI, 1971, 1983 a, b). Cependant au vu de ces différences significatives en 1965 et 1966, il est vraisemblable que la gradation ait déjà atteint un fort niveau dès 1962-1964. En revanche, comme pour les courbes standard, il n'est rien observé durant la période 1972 à 1976. Au Tilerga, on observe également un incident au cours de l'année 1971 qui ne peut être imputé à la processionnaire car sur les courbes standard il apparaît aussi bien sur les laricio que sur les maritimes. Il pourrait être du à un incendie ou à une action différée de la sécheresse de 1970. Les défoliations dues à la processionnaire ont donc causé dans les placettes observées en Corse des pertes daccroissement notables bien que moins importantes qu'au Ventoux : on n'y observe pas de cerne nul.

\subsection{Etude sur les accroissements individuels}

Cette analyse, compte tenu des données existantes arbre par arbre au PK 51, n'a pu être effectuée qu'à partir de 1972. Elle ne prend donc en compte que la seconde période d'attaque dont les conséquences sur l'accroissement ne sont apparues ni sur les courbes standard, ni dans les analyses en régression multiple. Le pourcentage d'inertie du nuage expliqué par les trois premiers axes est de 48,9 p. 100 pour le premier, 14,1 p. 100 pour le second et 12 p. 100 pour le troisième.

L'examen des corrélations avec les deux premiers axes (fig. 5) montre que le premier axe est très lié à la fois aux accroissements et aux populations de processionnaires; sur l'axe 2, les nombres de nids sont dans la partie négative de l'axe, les accroissements sont généralement dans la partie positive, ils sont très proches deux à deux si l'on considère les années consécutives 1972 et 1973, 1974 et 1975...). Il n’y a donc pas de différence d'accroissement entre les années paires et les années impaires bien que les attaques n'aient lieu qu'un an sur deux; ce fait a par ailleurs été confirmé par une analyse discriminante. Sur laxe 2, les accroissements s'opposent aux populations, en particulier au nombre de nids total et aux populations fortes de 1972, 1974 et 1976 ainsi quaux populations beaucoup plus faibles de 1982. Cette relation peut provenir des liaisons entre les populations elles-mêmes d'annće en annće par le phénomène de pin piège confirmé par Geri \& MiLlier (1985). C'est l'accroissement de 1978 qui s'oppose le plus aux attaques sur l’axe 2, il est situé après la période d'attaque de 1972 à 1976, cet antagonisme pourrait traduire un effet cumulé de l'infestation se répercutant sur cet accroissement.

En ce qui concerne les variables supplémentaires, la densité s'oppose à la fois au nombre de nids total et à un vecteur imaginaire «accroissement moyen». En effet, les peuplements denses sont moins attaqués du fait du comportement des adultes (Demolin, 1969 a) et de la dynamique des populations de la processionnaire (Geri, 1983 a), par ailleurs dans les boisements serrés, les arbres ont un accroissement radial moindre que dans des peuplements clairs. Les vecteurs circonférence et hauteur, très liés entre eux, sont orthogonaux aux nombres de nids en général et au nombre de nids total en particulier : la taille des arbres semble être indépendante des attaques subies.

La représentation des individus dans le premier plan principal (fig. 6 b) ainsi que leurs caractéristiques principales (fig. 6 a) confirme ces résultats. Sur l'axe 1, les arbres 1 et 4 s'opposent : l'arbre 4 forme les plus faibles accroissements et est très peu attaqué, l'arbre 1 possède les accroissements et le nombre de nids les plus forts. Sur 


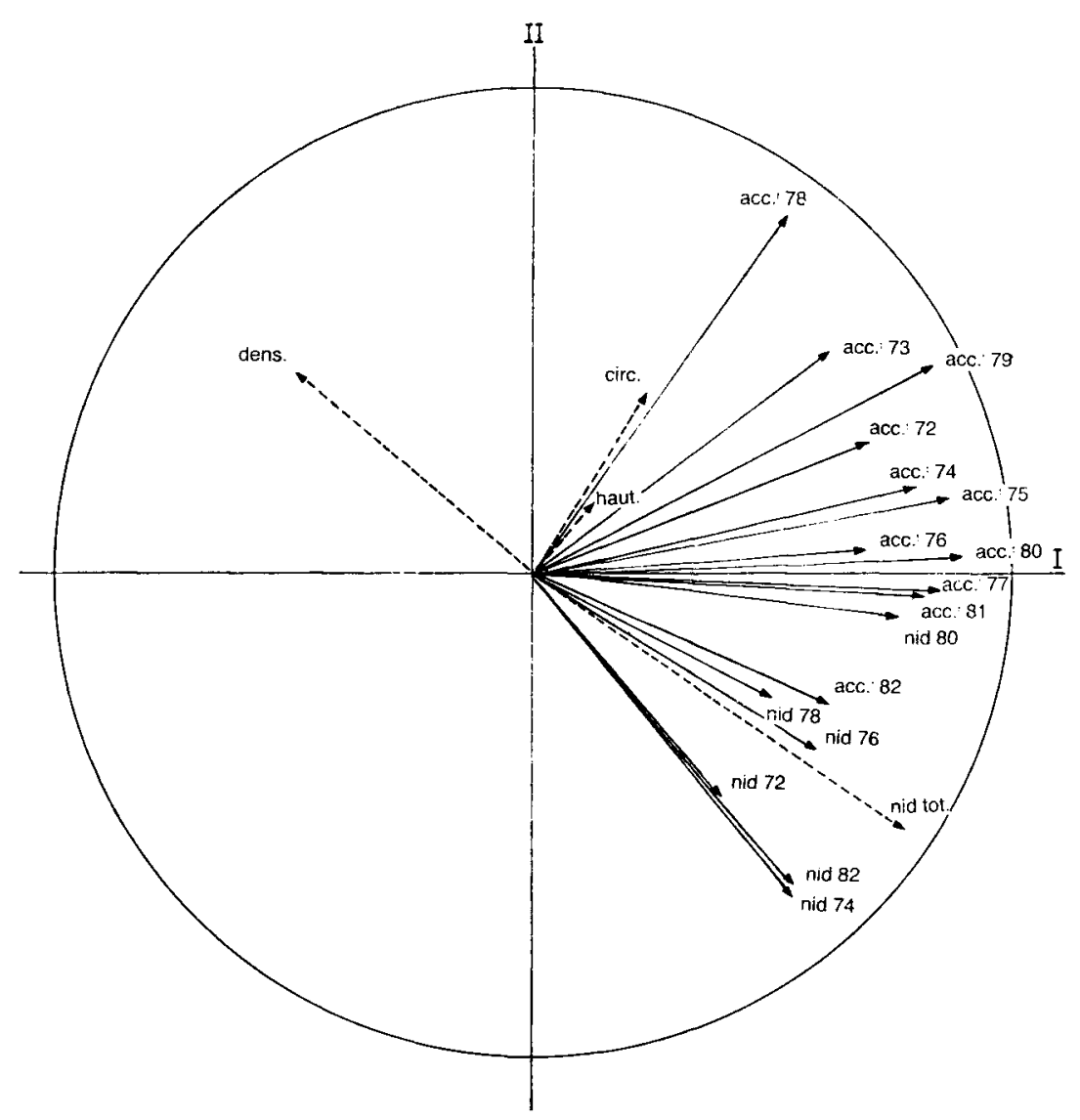

FIG. 5

Analyse en composantes principales obtenue à partir de la matrice $B$. Représentation des variables suivant leurs corrélations avec les deux premiers axes.

Principal components analysis obtained from $B$ matrix.

Display of variables according to their correlations with both first axes.

Variables supplémentaires :

dens : surface terrière de la placette situće autour de chaque arbre;

circ : circonférence ;

haut : hauteur ;

nid tot : nombre total de nids supportés par l'arbre de 1972 à 1982.

Additional variables:

dens : stand basal area around each tree;

circ : girth;

haut : height;

nid tot : total number of nests boren by the tree from 1972 to 1982. 


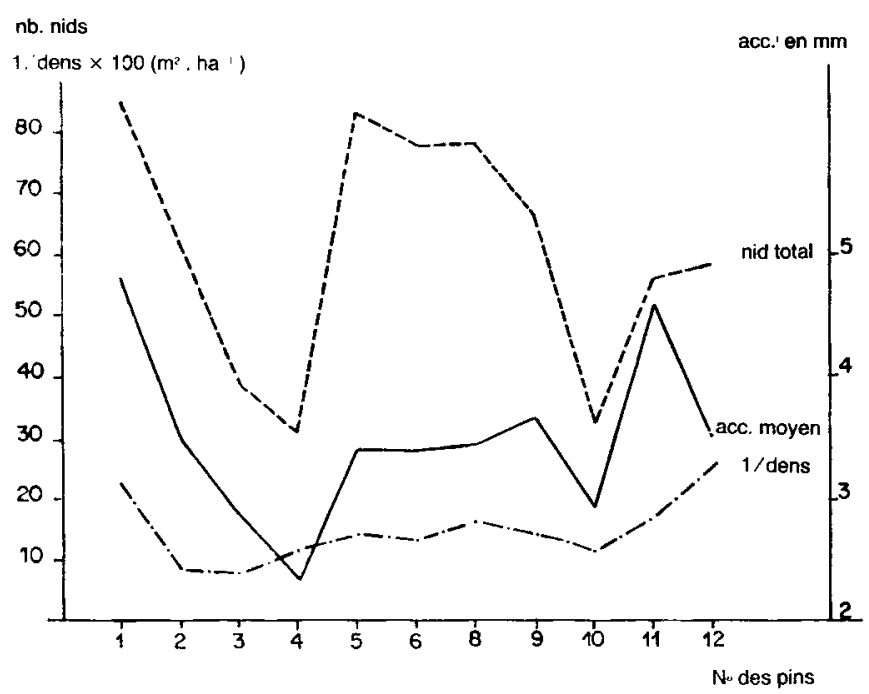

FIG. 6 a

Principales caractéristiques des onze pins du $P K 51$. Main characteristics of the 11 pines of PK 51 .

acc. moy. : moyenne des accroissements annuels de 1972 à 1982. acc. moy. : mean of the annual growth from 1972 to 1982.

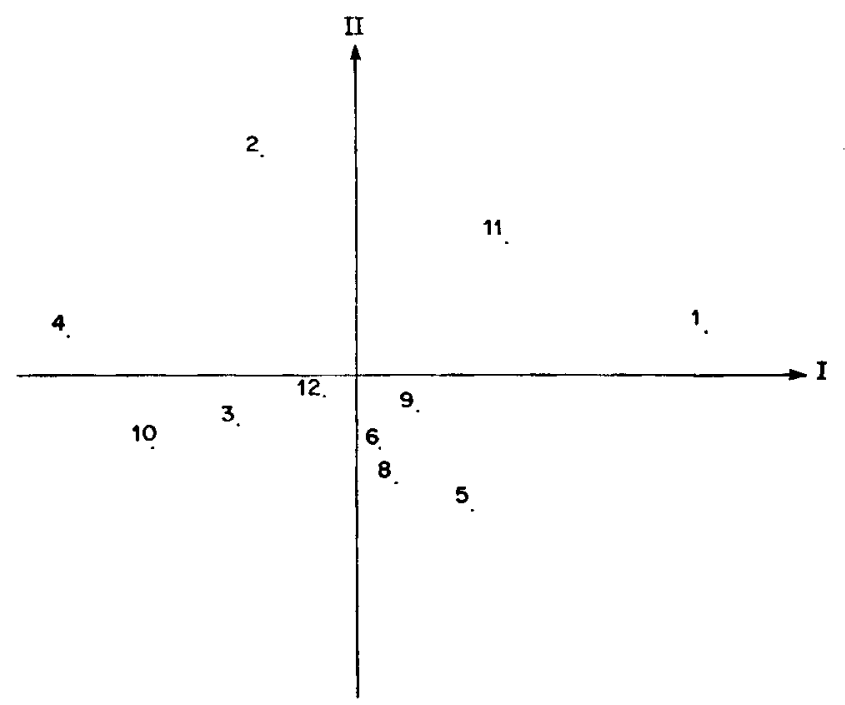

Fig. $6 \mathrm{~b}$

Représentation des onze pins dans le premier plan principal. Display of pines in the first principal plan. 
l'axe 2, s'opposent les pins 2 et 11 et les pins 5, 6 et 8 qui ont tous un accroissement moyen; mais les pins 2 et 11 ont un rapport accroissement sur nombre de nids fort tandis que les pins 5, 6 et 8 ont un rapport accroissement sur nombre de nids faible : l'axe 1 est un axe de fort accroissement et forte attaque alors que l'axe 2 traduit l'incidence des attaques sur les accroissements. Il faut souligner les faits suivants : si l'on considère les surfaces terrières, l'arbre 1 qui a la plus forte moyenne daccroissements et le plus grand nombre total de nids est un arbre très isolé, alors que les arbres 3, 4 et 10 qui possèdent les plus faibles accroissements et les plus faibles attaques appartiennent à des bosquets denses. L’arbre 1, du fait de son isolement, bénéficie d'une bonne croissance et est très attractif pour les processionnaires alors que les arbres 3, 4 et 10 gênés par leurs voisins, poussent mal et sont peu attractifs. Bien que les attaques de processionnaires aient eu même après 1972 un léger effet dépressif sur les accroissements, il semble que l'hétérogénéité du peuplement soit une cause importante de la difficulté de le mettre en évidence.

Dans ces peuplements naturels, les caractéristiques de l'arbre et de son environnement forestier, très hétérogènes d'un arbre à un autre jouent un rôle important sur la liaison entre les accroissements et les attaques de processionnaires; la densité des peuplements en particulier conditionne à la fois la croissance des pins et l'importance des attaques. Nous avons complété cette étude de l'incidence des attaques sur les accroissements depuis 1972 en considérant les indices de défoliation. Depuis 1972, aucun arbre n'a été défeuillé à plus de 50 p. 100. Or Rafes (1970) estime qu'en dessous de cette limite, l'effet d'une défoliation ne se fait pas sentir sur l'accroissement. WEвB et Wickman (1978) donnent deux explications à ce phénomène :

- en temps normal, les feuilles de la base ou du centre de la couronne n'atteignent pas l'intensité lumineuse pour laquelle la photosynthèse est maximale : en cas de défoliation partielle, ces feuilles reçoivent plus de lumière et ont une assimilation chlorophyllienne plus forte ;

- les aiguilles sont très exigeantes en carbone, la perte des aiguilles par défoliation entraîne une diminution importante de la demande en carbone. Ce phénomène peut être renforcé en Corse du fait de l'absence d'attaque tous les deux ans, ce qui laisse à l'arbre le temps de récupérer. Ceci expliquerait la faible incidence des attaques de 1972 à 1976 sur la croissance des arbres. Le seul effet est probablement un effet cumulatif dô à la défoliation répétée pendant plusieurs cycles de l'insecte que suggère l'analyse en composantes principales.

\subsection{Calcul de la perte d'accroissement}

\subsection{Pertes d'accroissement annuelles}

Les pertes d'accroissement radiales annuelles sont estimées : $P_{i}=y_{i}$ obs $-\hat{y}_{j}$ th où $\hat{y}_{\mathrm{i}}$ th est l'accroissement calculé en fonction du climat à partir de léquation de régression établie sur les pins maritimes. Elles sont représentées figure 7 comparativement à l'indice d'infestation moyen observé depuis 1968 dans les sous-zones où sont situćes ces placettes. Bien que seules les pertes d'accroissement de 1965 à 1968 soient significatives, il semble que l'ensemble de la période 1963-1969 ait subi des pertes de croissance. Après la gradation de 1974 à 1976, on note un fléchissement de la courbe à partir de 1977-1978 mais sans que cette chute devienne significative. On peut donc supposer que les infestations notées en 1966, 1968 (GERI, 1971, 1983 a) faisaient 

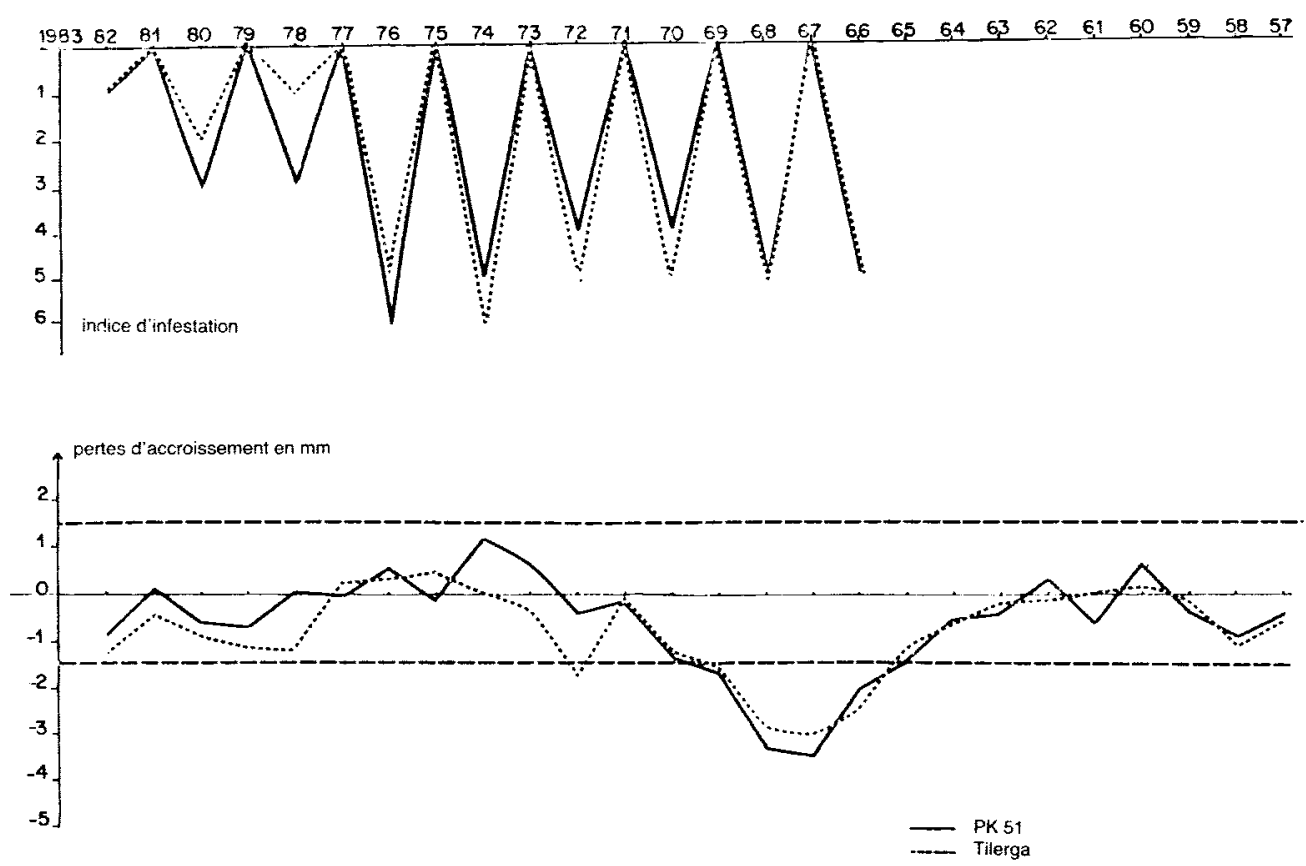

FIG. 7

Comparaison entre les pertes d'accroissement radial annuelles et les indices d'infestation dans la vallée du Niolo.

Comparison between annual radial growth losses and infestation indices in Niolo valley.

elles-mêmes suite à plusieurs années de très fortes infestations et que cette première a été beaucoup plus importante que celle observée en 1974-1976.

\subsection{Pertes d'accroissement sur plusieurs années}

La différence entre accroissements observés et accroissements estimés est significative sur les 26 années pour lesquelles nous disposons des données : on atteint en effectuant un calcul identique à celui du Ventoux 14,5 p. 100 pour le PK 51 et 19,2 p. 100 pour le Tilerga de perte d'accroissement pour toute cette période et respectivement 55,7 p. 100 et 53,3 p. 100 pendant la première gradation (années 1965 à 1968). Cette perte est importante et prouve que ni les intergradations ni l'absence d'attaques un an sur deux ne permettent à l'arbre d'éviter les conséquences des attaques de processionnaires.

\section{Importance économique}

Les pertes de production posent le problème de la nécessité et de la modalité des interventions. Des interventions directes peuvent être réalisées à partir de produits 
assez spécifiques comme le bacille Bacillus thuringiensis, spécifique des lépidoptères (Grison, 1970) et le Diflubenzuron qui intervient comme un inhibiteur de mues (Demolin \& Millet, 1981).

Ce type d'intervention peut se justifier du point de vue sanitaire par les urtications violentes provoquées par les poils de chenille, notamment en zone de fréquentation humaine importante (zones touristiques littorales...). D'un point de vue économique, on peut comparer le coût des traitements effectués à celui des pertes de production. Les pins noirs d'Autriche des peuplements du Ventoux sont vendus sur pied au prix moyen de $150 \mathrm{~F} / \mathrm{m}^{3}$ à l'automne 1983 , d'après leur hauteur dominante, ils appartiennent à la classe de fertilité 4 soit à 80 ans un accroissement courant de $3,1 \mathrm{~m}^{3} / \mathrm{ha} /$ an (OTTorini \& Toth, 1975), la forêt produit donc $150 \times 3,1$ $=465 \mathrm{~F} / \mathrm{ha} / \mathrm{an}$. Si on considère que la perte en volume au moins égale à la perte d'accroissement radial est de 30 p. 100 , on perd $465 \times 0,3=139,5 \mathrm{~F} / \mathrm{ha} / \mathrm{an}$, chiffre vraisemblablement sous-estimé du fait du traitement de 1979. Un traitement au Dimilin revient entre 200 et $250 \mathrm{~F} / \mathrm{ha}$, il devrait être répété plusieurs fois à intervalles de 3 ans environ pour éviter les réinfestations notamment à partir des individus en diapause, ce qui revient au maximum à $83 \mathrm{~F} / \mathrm{ha} / \mathrm{an}$ d'où un gain approximatif de $56 \mathrm{~F} / \mathrm{ha} / \mathrm{an}$. Un calcul similaire en Corse donne des résultats voisins.

\section{Conclusion}

Quel que soit le milieu étudié (plantation de pins noirs d'Autriche au Ventoux ou extension de la forêt dans d'anciennes terres de culture dans le Niolo), on trouve toujours à partir du moment où la gradation est suffisamment forte pour que le seuil de défoliation minimal soit dépassé, une perte d'accroissement radial non négligeable, que les périodes d'intergradation ne parviennent pas à tamponner.

Le problème de l'extrapolation de la perte d'accroissement radial à la perte en volume reste posé et sera évoqué dans un article ultérieur. Il semble néanmoins que la perte en volume soit toujours supćrieure ou égale à la perte sur le diamètre (Bouchon, communication personnelle). Les valeurs des pertes d'accroissement trouvées au Ventoux sont nettement supérieures à celles calculées par Bouchon et TOTH en 1971 au Ventoux et par Lemorne en 1977 dans les Landes qui étaient de lordre de 30 p. 100 pour les cinq années successives à l'attaque.

On peut difficilement comparer ces résultats avec ceux trouvés par LemorNe: dans les Landes où la situation est différente, les attaques se produisent rarement au même endroit, le problème du cumul des pertes d'accroissement d'une attaque à une autre ne se pose pas. Par ailleurs, Bouchov et Toth (1971) se sont toujours placés, pour les calculs, dans des hypothèses basses en particulier lorsque les causes des pertes de production étaient douteuses. Cependant, au Ventoux, les pertes de production ont probablement été sous-estimées dans tous les cas : comme Bouchon, nous avons supposé qu'en période hors attaque, les cernes observés indiquaient le potentiel d'accroissement et par ailleurs, nous avons dû ignorer la mortalité juvénile ainsi que le retard pris dans le jeune âge par les arbres attaqués.

Si des traitements chimiques sont justifiables à la fois d'un point de vue économique et d'un point de vue sanitaire, il est impossible d'apprécier actuellement les conséquences biologiques exactes de traitements répétés sur de grandes étendues. 
C'est pourquoi les traitements devraient surtout être limités aux zones de très forte infestation et l'on devrait plutôt, chaque fois que faire se peut, recourir à des mesures de type préventif : choix des essences et conduite des peuplements forestiers (PARDÉ, 1974). Seule la perte en quantité de bois formé a été étudiée, d'autres caractéristiques peuvent être modifićes à la suite des attaques de processionnaires, notamment la qualité du bois. Une ćtude est actuellement effectuée à ce sujet sur le matćriel de Corse au Centre National de la Recherche Forestière à l'INRA Nancy. Il est en effet probable que la connaissance exacte des conséquences des attaques de ravagcurs pour l'arbre doive allier dendrochronologie et xylochronologie.

Reģu en mai 1985. Accepté en juillet 1985.

\section{Remerciements}

Je tiens à remercier ici MM. BOuchon du CNRF Nancy et GERI de l'INRA Orléans pour leurs conseils et Jeur disponibilité ainsi que M. PINGUET du STEFCE à Avignon pour ses conseils et les renseignements météorologiques transmis. Mes remerciements vont également à la station de zoologie forestière de l'INRA Avignon et aux centres O.N.F. d'Avignon et de Bastia pour l'aide qu'il nous ont apporté dans la réalisation de ce travail.

\section{Summary}

Radial growth losses in some species of Pinus due to two forest defoliators $I$ - Case of the pine processionary moth in the Mediterranean area

The impact of defoliating carterpillars (Thaumetopoea pityocampa Schiff.) on radial growth was studied by dendrochronology on «Mont Ventoux》 on Austrian black pine (Pinus nigra Arn. ssp. nigricans Host.) and in Niolo valley in Corsica on corsican black pine (Pinus nigra Arn. ssp. laricio Poiret). Control-trees unattacked at study sites were Atlas cedar (Cedrus atlantica Manetti) at «Mont Ventoux» and cluster pine (Pinus pinaster Ait.) in Corsica. A dendroclimatological study of these species permitted us to remove the effect of climatic factors on radial growth in order to study the effects of defoliation.

At «Mont Ventoux», we could not detect a growth ring in 1968 in two plots just after the severe attacks of 1966 and 1967. A study of the last 25 years showed the followings :

1) radial growth has been more influenced by defoliation damage than by climate;

2) defoliation in the autumn of year $n-1$ or spring of year $n$ has an effect on radial growth of year $\mathrm{n}+1$;

3) the most heavily attacked plot showed a total reduction of 35 p. 100.

In Corsica, growth losses were 20 p. 100 for the 28 years studied but the attacks take place only every other year and are not comparable to the situation at «Ventoux ». Only the severe attacks of 1964 and 1966 affected tree growth, the 1974 and 1976 attacks had no effect. The degree of defoliation observed in 1974 and 1976 was below 50 p. 100 .

Key words: Pinus, thaumetopoea pityocampa, growth losses, dendrochronology.

\section{Références bibliographiques}

Bachacou J., Masson J.P., Millin:R C., 1981. Manuel de la Programmathèque statistique Amance. INRA, Département de Biométric, $400 \mathrm{p}$.

Bouchon J., Tотн J., 1971. Etudes préliminaires sur les pertes de production des pinèdes soumises aux attaques de Processionnaire du pin Thaumetopoea pityocampa Schiff. Ann. Sci. For., 28 (3), 323-340. 
Cadahia D., Insua A., 1970. Estimación de los daños producidos por Thaumetopoca pityocampa en las repoblaciones de Pinus radiata. OlLB. Coloquio de Teruel, 2-6 Noviembre, $14 \mathrm{p}$.

Cailliez F., Pages J.P., 1976. Introduction a l'Analyse des données, SMASH, Paris, 603 p.

Demolin G., 1969 a. Comportement des adultes de Thaumetopoea pityocampa Schiff. Dispersion spatiale. Importance écologique. Anr. Sci. For., 26 (1), 81-102.

Demolin G., 1969 b. Bioecologia de la Procesionaria del Pino. Incidencia de los factores climaticos, Bol. Serv. Plagas For., 12 (23), 9-24.

Demolin G., 1971. Incidences de quelques facteurs agissant sur le comportement social des chenilles pendant la période de procession de nymphose. Répercussion sur l'efficacité des parasites. Ann. Zool. Ecol. anim., 45, 33-56.

Demolin G., 1974. Incidence du climat sur les gradations de processionnaire du pin. Recherche d'éléments ou d'indices sur les potentialités négatives ou positives des populations. Prognose. Compte rendu de fin de contrat. D.G.R.S.T., 15 p.

Demolin G., Millet A., 1981. Essais insecticides contre la processionnaire du pin (Thaumetopoea Pityocampa Schiff). Action comparative des différentes spécialités commerciales : Bactospéine, Dipel, Thuricide et Dimilin. Ann. Sci. For. 1981, 38 (3), 389-404.

FritTs H.C., 1976. Tree Rings and climate, Academic Press, London, 505 p.

Geri C., 1971. Etude du niveau de population de Thametopoea pityocampa Schiff. dans la vallée du Niolo en Corse. Notes préliminaires sur les cycles biologiques 1965-1966 et 1967-1968. Ann. Zool. Ecol. anim., n" H.S., 2 (H.S.), 67-87.

GerI C., 1980. Etude sur les populations de processionnaire du pin Thaumetopoea pityocampa. Ecologia Mediterr., 6, 151-172.

Gerı C., 1983 a. Dynamique de la processionnaire du pin dans la valléc du Niolo en Corse au cours des cycles 1965-1966, 1967-1968 et 1969-1970. Rôles de certains caractères du milieu forestier. Ann. Sci. For., 40 (2), 123-156.

Geri C., 1983 b. Répartition et évolution des populations de processionnaire du pin dans les montagnes corses. I. Régimes d'apparition de l'insecte et dynamique des populations. Acta Oecol., Oecol. appl., 4 (3), 247-268.

Geri C., Millier C., 1985. Mesure des populations de processionnaire du pin (Thaumetopoea pityocampa Schitt, Lep. Thametopoeidae) au Mont Ventoux. Ann. Sci. For., 42 (2), 143-184.

Grison P., 1970. Lucha microbiologica contra la «procesionaria del pino». Bol. Serv. Plagas for., Madrid, 13 (26), 133-140.

JoLY R., 1959. Influence des défoliateurs forestiers sur l'accroissement. Rev. for. Fr.. 11, 775-784.

Kulman H.M., 1971. Effects of insect defoliation on growth and mortality of trees. Department of Entomology. University of Minesota.

LAURENT HervouËT N., 1984. Impact de la chenille processionnaire du pin sur l'accroissement radial du pin noir d'Autriche au Ventoux et du pin laricio en Corse : étude et mesure. Mémoire de fin d'études, E.N.I.T.E.F., 75 p.

Lemorne B., 1977. Contribution à la mesure des pertes de production causées par la chenille processionnaire du pin maritime dans les Landes. Ann. Sci. For., 34 (3), 205-214.

Munaut A.V., 1978. La dendrochronologie, une synthèse de ses méthodes et applications. Lejeunia n" 91,47 p.

Ottorini J.M., Toth J. Tables de production pour le pin noir d'Autriche dans le Sud-Est de la France. INRA-CNRF, Station de sylviculture et de production. Document $\mathrm{n}^{\circ} 75 / \mathrm{FM} / 04$. Distribution limitée. 
Polge H., Garkos S., 1971. Influence de défoliaisons sur la structure du bois de pin maritime. Ann. Sci. For., 28 (2), 195-206.

Parde J., 1974. Ravageurs, lutte intégrée et sylviculture. Rev. For. Fr., 6, 419-426.

Rafes P.M., 1970. Ecological studies. 1. Analysis of Temperate Forest Ecosystems. Estimation of the effects of Phytophagous insects on Forest production. Ed. by David E. Reichle, Berlin, Heidelberg, New York, 100-106.

Schwartz. D., 1969. Méthodes statistiques' à l'usage des médecins et des biologistes, 3* édition, Flammarion, Médecine, Sciences, Paris, $260 \mathrm{p}$.

TRENard Y., 1982. Making wood speaking introduction to dendrochronology. Forestry Abstracts. Déc., 43 (12).

WEBB W.L., 1978. Effects of defoliation and tree energetics. In : «The Douglas Fir Tussock Moth : a synthesis ». M.H. Brookes, R.W. Stark, R.W. Campbell edit. Washington, $77-81$

Wickman B.E., 1978. Tree Injury. In : «The Douglas Fir Tussock Moth : a synthesis». M.H. Brookes, R.W. Stark, R.W. Campbell edit. Washington, 66-77. 